Erstveröffentlichung:

Lorenz, Astrid, Ordnung und Wandel des Grundgesetzes als Ergebnis

des Wechselspiels von Politik und Recht, in: dies./Reutter, Werner

(Hrsg.), Ordnung und Wandel als Herausforderungen für Staat und

Gesellschaft, Opladen 2009, S. 205-232.

Astrid Lorenz

\title{
Ordnung und Wandel des Grundgesetzes als Ergebnis des Wechselspiels von Politik und Recht
}

\section{Einleitung}

In Demokratien wie der Bundesrepublik befinden sich Verfassungen in einem beständigen Spannungsverhältnis zwischen konstitutionalistischem „Ordnen“ und demokratischem „Gestalten“. Im Sinne des Konstitutionalismus sind sie einerseits darauf angelegt, dem Staat Grenzen zu setzen und staatliches beziehungsweise politisches Handeln dauerhaft zu regeln. Nach den Diktaturerfahrungen des 20. Jahrhunderts wurde den obersten Staatsorganen in Deutschland wie in vielen anderen europäischen Staaten ein starkes Verfassungsgericht zur Seite gestellt. Es soll als oberster Hüter der Verfassung verhindern, dass einfaches Recht gegen Verfassungsrecht verstößt und dass die potentiellen Normverletzer identisch sind mit denjenigen, die die Normen aus eigenem Recht schaffen und deren Einhaltung garantieren (Isensee 1995a: 50f.). Andererseits enthält heute jede Staatsverfassung Regeln zur eigenen Änderung, also Möglichkeiten des gestalterischen Eingriffs in sie selbst. Solche Änderungen bedürfen größerer Mehrheiten als „gewöhnliche“ Gesetze - in der Bundesrepublik einer Zweidrittelmehrheit in Bundestag und Bundesrat. Anders gesagt: Verfassungen, auch das Grundgesetz, sind Wandlungsimpulsen ausgesetzt, die sich in Form von Textmodifikationen, verfassungsgerichtlichen Interpretationen oder veränderten Routinen und Konventionen äußern können.

Im Gegensatz zur „von oben“ gesetzten Ordnung autoritärer Systeme wie der DDR, die Wandel zwar zulassen oder zulassen müssen, aber eben nur ,von oben" kontrolliert und gesteuert (Glaeßner 1977, 1989), setzen liberal-demokratische Systeme der Gestaltung der politischen Ordnung andere Grenzen. Verfassungen bedürfen hier der demokratischen Rechtfertigung, sprich: der Legitimation. Legitimität wird einerseits, wie bemerkt, durch besondere Verfahrensvorschriften gewährleistet, andererseits müssen die Normen inhaltlich Anerkennung finden. Initiatoren von Verfassungsänderungen müssen also die Öffentlichkeit und die politische Aktivbürgerschaft von der Sinnhaftigkeit ihres Vorhabens überzeugen. Auch das Bundesverfassungsgericht kann, wenngleich nicht mit Initiativrecht ausgestattet, normschöpfend agieren und damit Politik (zumindest vorübergehend) ,machen“. 
Ordnung und Wandel von Verfassungen sind insofern komplex, als sich beide gegenseitig bedingen und durchdringen. Soll Verfassung nicht nur in alt-konstitutionalistischer Manier Politik und Staat einhegen und begrenzen, sondern Letzteren auch Ziele und Orientierung verleihen, im Idealfall sogar gesellschaftliche Rahmenbedingungen adäquat verarbeiten, dann kann sie nicht als bloß statisches Normengefüge begriffen und interpretiert werden. Vielmehr müssen unter von der Verfassung selbst gesetzten Bedingungen Änderungen möglich sein. In diesem dialektischen Spannungsverhältnis, in dem Wandel eine spezifische verfassungsrechtlich begründete Ordnung voraussetzt und Verfassung dauerhaft nur Akzeptanz finden kann, wenn sie Wandel verfassungskonform verarbeitet, bewegt sich die Verfassungspolitik der Bundesrepublik Deutschland.

Der vorliegende Aufsatz geht der Frage nach, inwieweit dieses Wechselverhältnis von Politik und Recht die Entwicklung des Grundgesetzes geprägt hat. Welche auf Ordnung angelegte Normen wurden verändert? Lassen sich Trends des gestalterischen Wandels der Verfassung erkennen? Um diese Fragen beantworten zu können, skizziert der Beitrag zunächst die zentralen Ordnungsprinzipien des Grundgesetzes, wie sie 1948/49 gefasst wurden. ${ }^{1}$ Im zweiten und dritten Teil schildert er die Charakteristika der Verfassungsänderungen, die entweder durch Gesetz oder infolge Rechtsprechung des Bundesverfassungsgerichts stattfanden. Abschließend resümiert der Aufsatz die Reichweite des Verfassungswandels, diskutiert dessen Legitimation und die Fähigkeit des Grundgesetzes, politisches Handeln zu ordnen.

\section{Ordnungsprinzipien der Verfassung 1949: Kein echter Konsens, keine Euphorie}

Niemand konnte 1949 vorhersehen, dass das Grundgesetz eine Erfolgsgeschichte erleben würde. Die Erarbeitung der Verfassung wurde nicht von den deutschen Parteien vorangetrieben, sondern von den Alliierten, die im Herbst 1948 die Ministerpräsidenten der Länder damit beauftragten. Für die SPD wandte sich Kurt Schumacher gegen alle Regelungen, die „Spannung und Spaltung" in Deutschland vorantrieben, und kritisierte noch kurz vor der Verabschiedung der Verfassung: „Man kann eben nicht ein Grundgesetz auf Kommando der Besatzungsmächte schaffen. Selbst in einem von ihnen gesteckten Rahmen muss eine Freiheit in der Entscheidung und der Willens-

1 Es geht also um die Ursachen und Mechanismen institutionellen Wandels, der nur nachzuweisen ist, wenn eine „Normalnull“ als Vergleichsmaß festgelegt ist. Der Vergleich mit 1949 impliziert nicht, dass die heutigen oder künftigen Verfassungsnormen noch den Intentionen der Verfassungsgeber von 1948/49 entsprechen müssen oder sollen. Diese sind bei der Auslegung von Verfassungsnormen nur einer von mehreren möglichen Bezugspunkten und genießen nicht per se eine höhere Dignität. 
bildung vorhanden sein. Man kann nicht vom deutschen Volke verlangen, dass es alles das auf alliierten Wunsch für richtig erklärt, von dem es doch weiß, dass es falsch ist und die deutsche Staatswerdung nur hindert". Auch der Präsident des Parlamentarischen Rates, Konrad Adenauer (CDU), beklagte sich über die „Gängelei“ der West-Alliierten (Schwind et al. 1999: 265).

Dennoch akzeptierten die Ministerpräsidenten den Auftrag der Alliierten zur Verfassungsgebung. Das Grundgesetz knüpfte wie die ersten westlichen Landesverfassungen - und wie nach ihm die (erste) Verfassung der DDR vom 7. Oktober 1949 - an die Weimarer Reichsverfassung an (Mommsen 1998; Kleßmann 1991). Wichtige verfassungsrechtliche Strukturprinzipien, so das Republik-, das Demokratie-, das Bundesstaats- und das Rechtsstaatsprinzip, belegen dies. Die Vorbildwirkung der Weimarer Reichsverfassung ist jedoch nicht absolut zu verstehen. Sie selbst wurde ja (ähnlich wie die Verfassungen der späteren DDR) nicht nur durch verfassungsdurchbrechende Gesetze, Verordnungen und eine verfassungsfeindliche politische Praxis ausgehöhlt, sondern enthielt bereits widersprüchliche Verfassungsgrundsätze (Kleßmann 1991: 208). Solche inhärenten Widersprüche sollten im Grundgesetz vermieden werden, ebenso alle Regeln, die für das Scheitern der ersten deutschen Demokratie verantwortlich gemacht wurden. Alle wollten also „Lehren aus Weimar“ ziehen. Wie das geschehen sollte, war jedoch umstritten.

Einig waren sich die großen Parteien in ihrer Befürwortung des Grundrechtekatalogs (inklusive des Verbots der Todesstrafe) und eines starken Parlaments bei weitgehendem Ausschluss direktdemokratischer Elemente aus dem Grundgesetz. Politischer Dissens existierte nicht nur hinsichtlich der strategischen Wirkung, die von der Verabschiedung einer Verfassung ausgehen würde, sondern auch hinsichtlich ihrer Inhalte, so der Funktion des Staatsoberhauptes, der zweiten Parlamentskammer (Bundesrat oder Senat), des Föderalismusmodells, der Verteilung der Steuern zwischen Bund und Ländern, des Verhältnisses von Kirche und Staat und des „Elternrechts“ zur Bestimmung der religiösen Erziehung. In diesen Fragen gelang es teils nur mithilfe interfraktioneller Gesprächskreise außerhalb der Geschäftsordnung, Kompromisse zu finden (Glaeßner 2006: 290ff.; Feldkamp 1998; Pfetsch 1990; Niclauß 1998). Die Finanzverfassung konnte angesichts gravierender Differenzen nur ,unter massivem politischem Druck“ entwickelt werden, und einer der beiden zentralen Artikel (106 GG) durfte gemäß dem Verfassungstext durch einfaches Gesetz bis Ende 1952, dann noch zweimal verlängert bis Ende 1955, geändert werden, um überhaupt zu einer Normierung zu kommen (Renzsch 1991: 69).

Die bedeutendsten Neuerungen des Grundgesetzes gegenüber der Weimarer Verfassung bestanden: im Bekenntnis zu unverletzlichen und unveräußerlichen Menschenrechten (Art. 1 GG), in der Bindung aller drei Gewalten an die im GG aufgeführten Grundrechte (Art. 1 Abs. 3 GG), in der RechtsschutzGeneralklausel (Art. 19 Abs. 4 GG), in der Festschreibung der ,freiheitlichen 
demokratischen Grundordnung“" (Art. 18, 21 Abs. 2, 91 Abs. 1 GG) inklusive Recht auf Opposition sowie der Parteien als Mitgestalter der politischen Willensbildung des Volkes (Art. $21 \mathrm{GG}$ ), in der weitreichenden Machtbegrenzung des indirekt gewählten Bundespräsidenten (Art. 54-61, 63-67, 67-68, 81 GG), in der Wahl des Bundeskanzlers durch den Bundestag (Art. 63 GG), im eingeschränkten Recht der Parlamentsauflösung (Art. 63, 68 GG), in der Einführung des auf die Person des Bundeskanzlers beschränkten konstruktiven Misstrauensvotums (Art. 67 GG), in der Gliederung des Bundesstaates in Länder, in deren Mitwirkung in der Bundesgesetzgebung durch den Bundesrat (Art. 50, 78 und 79 Abs. 3 GG) sowie in der Errichtung eines mit weitreichenden Kompetenzen ausgestatteten Bundesverfassungsgerichtes als letztinstanzlich entscheidendem Interpreten der Verfassung (Art. 92 bis 94 GG). Wesentliche Unterschiede bestanden im fast vollständigen Verzicht auf Volksbegehren und Volksentscheid und - bis 1969 - auf Notstandsbestimmungen entsprechend Art. 48 der Weimarer Verfassung (Steffani 1979: 87f.) sowie in der starken Stellung des Bundeskanzlers im politischen System.

Das Grundgesetz sollte - im Gegensatz zum Wunsch der Alliierten - lediglich Provisorium sein und eine Vollverfassung erst verabschiedet werden, wenn die deutsche Teilung überwunden war. Es stellte einen Kompromiss der Volksparteien CDU und SPD dar, die die Verhandlungen auf dem Herrenchiemseer Konvent und im Parlamentarischen Rat dominierten. Sie nahmen das Grundgesetz letztlich nicht deshalb an, weil sie die von konkreten Verfassungsnormen überzeugt waren, sondern weil sie die Notwendigkeit der Verfassungsgebung akzeptierten. Carlo Schmid (SPD) formulierte dies in der dritten Lesung des Grundgesetzes so: Man handele „unter dem Gesetz der Verantwortung vor dem Volke“ (Schwind et al. 1999: 265). Nahezu alle Vertreter der kleinen Parteien CSU (außer Mayr, Schlör), DP, Zentrum, KPD stimmten gegen den Verfassungsentwurf, nur die FDP dafür. Die CSU lehnte insbesondere die Vorherrschaft des Bundes ab, die sich im Grundsatz „Bundesrecht bricht Landesrecht“" (Art. 31 GG) äußerte, und wollte den Ländern deutlich größere Rechte einräumen. Diesen waren nach intensiven Verhandlungen die Ausführung der Gesetze, die innere Verwaltung, das Versorgungswesen, bestimmte Politikfelder und die Mitwirkung in der Bundesgesetzgebung zugestanden worden. Dennoch gab es Unzufriedenheit, etwa gegen die Bestimmungen zur Länderneugliederung (Art. 24 und 25 GG) in den badischen Teilen des neu zu gründenden Südweststaats.

Auch wenn es übertrieben wäre, von einem Oktroi der Alliierten zu sprechen, so bleibt doch festzuhalten, dass es ohne sie das Grundgesetz nicht zu diesem Zeitpunkt und nicht in dieser Form gegeben hätte. Der im Februar 1949 konturierte Verfassungsentwurf musste nach ihren Einwänden betreffend Finanzverwaltung, Gesetzgebungskompetenzen oder den Status Berlins revidiert werden, damit er überhaupt angenommen werden konnte (W. Benz 2005). Besonders im Hinblick auf den betonten provisorischen Charakter und den damit begründeten Verzicht auf eine Volksabstimmung setzten sich aber 
die deutschen Parteien durch. Das Grundgesetz wurde also nicht direkt vom Volke legitimiert, sondern lediglich vom Parlamentarischen Rat, der aus Abgeordneten der Landtage zusammengesetzt war, sowie nach der Genehmigung durch die Militärgouverneure von den Volksvertretungen der westdeutschen Länder angenommen. (Nur Bayern ratifizierte das Grundgesetz nie, akzeptierte aber die Mehrheitsentscheidung.) Generell war 1948/49 „das Mißtrauen der Ministerpräsidenten und der ersten gewählten Parlamentarier gegenüber der Bevölkerung sehr groß (...) Es herrschte das Gefühl vor, dieses Land habe keine Demokraten und sei von Demokratie nicht überzeugt" (Merseburger 1998). Nicht zu Unrecht, wenn man die empirischen Untersuchungen zur politischen Kultur der unmittelbaren und späteren Nachkriegszeit betrachtet (Merritt/Merritt 1970; Almond/Verba 1963).

Als zentrale Ordnungsprinzipien des politischen Systems fixierte das Grundgesetz: die Republik, die parlamentarische Demokratie, Rechtsstaatlichkeit, Sozialstaatlichkeit, eingeschränkte Souveränität sowie den Bundesstaat mit seiner spezifischen Kompetenzverteilung nach Staatsfunktionen, die ein kooperatives Miteinander (oder auch wechselseitige Abhängigkeiten) erfordert. Charakteristisch war zudem der bedeutende Grundrechtsschutz. Diese Prinzipien wurden unter „Ewigkeitsgarantie“ gestellt (Art. 79 Abs. 3 GG). Wieviel Raum für Wandel ließ diese verfassungsrechtliche Konzeption?

\section{Explizite Änderungen des Grundgesetzes: Häufig und vorrangig selbstreferenziell ${ }^{2}$}

Schon bald nach Verabschiedung des Grundgesetzes wurden erste Änderungen vorgenommen. Was zunächst noch als Nachbesserung und Ergänzung mit Blick auf die Hintergründe der Verfassungsgebung zu deuten war, setzte sich fort. Bis heute wurde der Text des Grundgesetzes 52mal geändert, ergänzt oder - seltener - gekürzt. Im Vergleich der Änderungsraten gehört die Bundesrepublik damit zu jenen Demokratien, die ihre Verfassung am häufigsten änderten. Dies steht im Kontrast zur üblichen Wahrnehmung Deutschlands als resistent gegenüber Wandel. Insgesamt wurden von den 192 aktuellen Grundgesetzartikeln (mit Präambel und Anhang) nur 85 (44 Prozent) noch nie verändert; 60 Artikel (31 Prozent) wurden hingegen einmal modifiziert oder ergänzt, 30 (16 Prozent) zweimal, weitere 17 (9 Prozent) sogar dreimal oder noch häufiger. Die meisten dieser Änderungen waren nicht auf weitreichende Reformen des politischen Systems angelegt, aber dies gilt für alle etablierten Demokratien (Lorenz 2008).

Die verabschiedeten Verfassungsänderungen wurden zu etwa gleichen Anteilen von den Bundesregierungen sowie von den Bundestagsfraktionen

2 Das Manuskript wurde Ende 2008 abgeschlossen. Die Entwicklungen um die Föderalismusreform II sind daher nicht erfasst. 
formal angestoßen. Initiativen der Bundesregierung erfolgten seltener, waren aber erfolgreicher. Gesetzentwürfe der Fraktionen traten zwar in wesentlich größerer Zahl auf, waren aber - wenig überraschend - eher dann erfolgreich, wenn sie bereits Lager übergreifend eingebracht wurden. In den ersten Jahrzehnten bundesrepublikanischer Verfassungsänderungen geschah dies oft nach informellen Vorabsprachen, in jüngerer Zeit haben solche fraktionsübergreifenden Initiativen oft bereits eine „Drucksachen-Vorgeschichte“: Sie wurden im Zuge von Ausschussverhandlungen zum selben oder auch zu anderen (von einer einzelnen Fraktion initiierten) Gesetzgebungsvorgängen eingebracht. Teils, wie beim Staatsziel Umweltschutz, gab es vor dem letztlich erfolgreichen Entwurf eine ganze Reihe nicht verabschiedeter Initiativen.

Der Bundesrat initiierte nur etwa fünf Prozent der verabschiedeten Änderungen und brachte verschwindend selten eine Verfassungsänderungsinitiative formal zu Fall. Dies kann zum einen dadurch erklärt werden, dass das Verfassungsrecht ihm nicht die Rolle eines proaktiv gestaltenden Bundesorgans, sondern des Mitgestalters der Bundesgesetzgebung zugewiesen hat. Die zurückhaltende Rolle des Bundesrats ist zum anderen dadurch zu erklären, dass die Landesexekutiven angesichts der oft inhomogenen parteipolitischen $\mathrm{Zu}$ sammensetzung des Bundesrates informelle Aushandlungskanäle bevorzugen, um politische Initiativen anzuregen.

Deutlich zeigen sich Themenaffinitäten der verschiedenen Initiatoren, die sich gemäß den geschilderten Initiativmustern und Erfolgsraten im Output der Verfassungsänderungen spiegeln. Am häufigsten wurde der Verfassungstext im Hinblick auf die Kompetenzverteilung zwischen Bund und Ländern, die Finanzverfassung, Belange der Justiz und Regelungen zum politischen Prozess, z.B. zum Gesetzgebungsverfahren, geändert (Tabelle 1; Lorenz 2007; Busch 1999). Davon stieß die Bundesregierung vor allem die Änderungen an, die sich auf die Staatsorganisation und konkrete Vorgaben für Politiken bezogen, darunter die Außen-, Verteidigungs- und Europapolitik.

Auf Initiativen des Bundestags lassen sich die meisten Grundgesetzänderungen zu Bürgerrechts- und Beteiligungsfragen zurückführen, die insgesamt aber einen deutlich geringeren Anteil der Änderungen ausmachen, weil es in diesen Wertefragen und Konflikten über die verfassungsrechtliche Verarbeitung gesellschaftlichen Wandels schwerer gelingt, den für die Bereitstellung der Zweidrittelmehrheit notwendigen Konsens zu erzielen. Dass die Fraktionen hier dennoch aktiv bleiben, zeugt von einer hohen Motivation ,in der Sache" und von der Bereitschaft, auch auf der Klaviatur symbolischer Politik zu spielen. Die großen Verfassungskonflikte ${ }^{3}$ mündeten insgesamt aber selten in

3 Dies waren die Konflikte um Parteiverbote 1952-1956 (BVerfGE: E 2,1 5, 85), um die Wiederbewaffnung, Notstandsgesetze, den Radikalenerlass bzw. die freiheitlich-demokratische Grundordnung (BVerfGE 39, 334), die Ostverträge (1972: BVerfGE 36,1), den Schwangerschaftsabbruch (1975, 1993: BVerfGE 39,1; 88, 203; 98, 265), Volkszählung (1983: E 65,1), Sitzblockaden (1986, 1995: E 73, 206; 92, 1), den Vertrag von Maastricht (1993: E 89, 155), 
expliziten Textänderungen, sondern wurden überwiegend unterhalb der Verfassung bzw. vor dem Bundesverfassungsgericht ausgetragen. Der größte Posten erfolgreicher Verfassungsänderungsentwürfe aus dem Bundestag betraf die Gesetzgebung und Kompetenzen von Bund und Ländern (Lorenz 2007).

Tabelle 1: Gegenstände von Grundgesetzänderungen, 1949-2006

\begin{tabular}{lcclcc}
\hline Änderungsgegenstand & Häufigkeit* & & Änderungsgegenstand & Häufigkeit* \\
\cline { 1 - 2 } Konkurrierende Gesetzgebung & 18 & & Notstandsrecht & 2 \\
Finanzen/Haushalt & 9 & & Petition & 2 \\
Steuern & 9 & & Wiedervereinigung & 2 \\
Justiz/Strafverfolgung & 7 & & Antidiskriminierung & 1 \\
Wahlrecht & 5 & & Asylrecht & 1 \\
Gesetzgebungsprocedere & 4 & & Beamtentum/Besoldung & 1 \\
Gliederung Bundesgebiet & 4 & & Europäische Integration & 1 \\
Lastenausgleich & 4 & & Grundrechte & 1 \\
Übergangsregelungen & 4 & & Internationale Verträge & 1 \\
Vertriebene/Kriegsfolgen & 4 & & Kernenergie & 1 \\
Privatisierung & 3 & & Parteienfinanzierung & 1 \\
Streitkräfte/Verteidigung & 3 & & Staatsverwaltung & 1 \\
Umwelt/Tierschutz & 3 & & Wehrpflicht & 1 \\
Innere Sicherheit & 2 & & Gesamt & 95 \\
\hline
\end{tabular}

* Gibt an, wie viele Grundgesetzänderungsgesetze den Gegenstandsbereich berührten. Da die Gesetze mehrere Bereiche berühren können, ist die Gesamtzahl nicht identisch mit der Gesamtzahl verabschiedeter Gesetze.

Quelle: Grundgesetzänderungsgesetze; eigene Zusammenstellung.

Schaubild 1: Grundgesetzänderungen nach Jahren, 1949 bis 2006

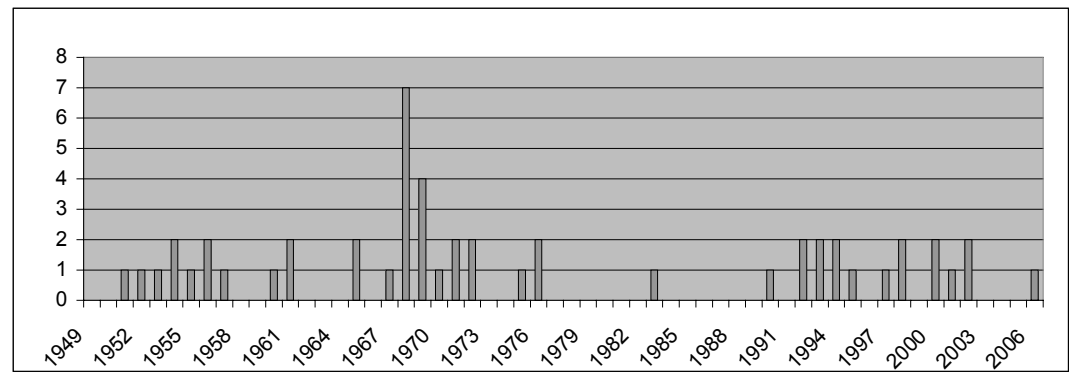

Anmerkung: Die Grundgesetzänderungen sind erfasst nach dem Jahr der Verabschiedung im Bundestag.

Quelle: Grundgesetzänderungsgesetze.

,Soldaten sind Mörder‘ (1994-95: E 93, 266) und religiöse Symbolik im öffentlichen Raum (BVerfGE 93,1). Mayer 2002. 
Ähnlich wie in anderen etablierten Demokratien traten Verfassungsänderungen (ebenso wie Änderungsinitiativen) in Deutschland zyklisch auf. Diese Zyklen lassen sich nur teilweise auf gesellschaftlichen beziehungsweise politischen Änderungsdruck zurückführen. Ein Großteil der Verfassungsänderungen während der Konjunkturwipfel steht allenfalls in einem indirekten $\mathrm{Zu}$ sammenhang mit dem entsprechenden gesellschaftspolitischen Kontext. So hatten die Änderungen nach 1990 mit der deutschen Einheit inhaltlich relativ wenig zu tun. Besonders dann, wenn die Verfassung kurz zuvor geändert wurde, nahmen die politischen Akteure die Verfassung nicht als dauerhaft „Zwingendes Recht“"wahr, das „,nicht [zur] Disposition der Beteiligten“ steht (Isensee 1995b: 27), sondern als veränderbar (Schaubild 1).

Die Themenaffinitäten, die große Häufigkeit von Änderungen bei einem von spezifischen Ereignissen und parteipolitischen Konstellationen relativ unabhängigen Konjunkturverlauf lassen es gerechtfertigt erscheinen, den expliziten Wandel des Grundgesetzes als Ergebnis vorrangig selbstreferenziellen Handelns politischer Eliten zu bezeichnen. Die Verfassungspolitik reagierte zwar auch auf Änderungen der gesellschaftlichen Rahmenbedingungen (u.a. Wertewandel, Problemlagen), aber verzögert, sehr selektiv und verfügte über Ausgestaltungsspielräume. Bei Themen, die ihren Alltag und sie selbst konkret betrafen, erzielten sie weitaus häufiger Einigungen. Es herrscht also einerseits in Bezug auf die Verfassungsnormen, die die Beteiligten selbst betreffen, durchaus Dynamik. Andererseits lässt sich eine Kontinuität der Grundnormen feststellen, womit der Idee Rechnung getragen wird, dass sich in Verfassungen unstrittige, integrierende Regelungen finden und wenig Zeitgeist. Denn bei aller Häufigkeit der Grundgesetzänderungen wurden die zentralen Ordnungsprinzipien selten berührt. Das Demokratie- und Republikprinzip, Gewaltenteilung, Föderalismus, Rechts- und Sozialstaatlichkeit werden selbst von der parlamentarischen Opposition nicht infrage gestellt, und gegen politische Minderheiten, die in diesen Punkten nicht auf dem Boden der Verfassung stehen, schützt sich der Staat im Sinne der ,wehrhaften Demokratie“.

Politisch wiederkehrende und umstrittene Themen betrafen: die Kompetenzverteilung im Bundesstaat, Grundrechtsänderungen und die Ergänzung der Offenheit für die europäische Integration. Sie werden im Folgenden umrissen.

\section{Der Bundesstaat - die ewige verfassungspolitische „Baustelle“}

Die „Unitarisierung“ der Bundesrepublik äußerte sich darin, dass immer mehr Materien, die ursprünglich in den Kompetenzbereich der Länder fielen, durch den Bund geregelt wurden (Abromeit 1992). Die Länder stimmten dieser Kompetenzwanderung nach gängiger Meinung deswegen zu, weil sie im Gegenzug via Bundesrat in der Bundesgesetzgebung mitwirken konnten. Der 
reale Anteil von Zustimmungsgesetzen, der ohnehin von Anfang an höher ausfiel, als die Verfasser des Grundgesetzes angenommen hatten, stieg denn auch sukzessive an. Bereits 1976 stellte die Enquête-Kommission „Verfassungsreform“ fest, dass die Gesetzgebung „,ganz überwiegend zur Bundessache geworden ist" (BT-Drs. 7/5924: 126). Ausgelöst wurde die Zustimmungsbedürftigkeit bei der Hälfte der entsprechend deklarierten Gesetze durch Art. 84 Abs. 1 GG a.F., weil das jeweilige Bundesgesetz zugleich die Einrichtung von Behörden oder das Verwaltungsverfahren bei der Gesetzesausführung regelte, also in den ursprünglich genuinen Kompetenzbereich der Länder - die Gesetzesausführung - eingriff (Dästner 2001). Es gab aber auch Argumente mit Blick auf das Gemeinwohl und die Entscheidungseffizienz für diese Entwicklung. Den hohen Wert einer Einheitlichkeit der Lebensverhältnisse der Bürger stützte das Bundesverfassungsgericht in seiner frühen Rechtsprechung mit der so genannten Einheits- oder Unteilbarkeitstheorie (Burkhart/Manow 2006; Scharpf 2006; Korioth 2006). Durchaus plausibel sind auch die Einsparung von Kosten und zeitaufwendigen Abstimmungen bei einer zentralen Regelung beziehungsweise Verwaltung einer Materie.

Verteilungskonflikte zwischen den Ländern, strukturelle Finanzprobleme und knappe Kassen änderten in den 1990er Jahren die Problemwahrnehmung; Wettbewerb schien eine Möglichkeit, die Schwierigkeiten zu lösen. Verstärkt wurde darauf hingewiesen, dass die ,wichtigste Rechtfertigung für ein föderales System (...) gerade in der Bewahrung und Ermöglichung von regionaler Vielfalt" bestehe. Deswegen sollten die Gemeinschaftsaufgaben eingeschmolzen und die Gestaltungsmöglichkeiten der Länder gestärkt werden. Gestaltungs- anstelle Verbundföderalismus und Abbau der Mischfinanzierungen lauteten die Ziele (Grimm 2005: 2). Dass diese Argumentation mehrheitsfähig wurde, hing mit den politischen Kräfteverhältnissen zusammen: Als die Mehrheitsverhältnisse in Bundestag und Bundesrat den Verhandlungsbedarf erhöhten, stießen Bayern und Baden-Württemberg 1996 eine öffentliche Debatte über die, so die Bewertung, mangelnde internationale Wettbewerbsfähigkeit des deutschen Föderalismus an. Konservative und Sozialdemokraten schoben sich gegenseitig den "Schwarzen Peter“ zu. Der Machtwechsel zu Rot-Grün auf Bundesebene 1998 änderte an dem Streit um die Zukunft des deutschen Föderalismus nichts, weil auch die neue Bundesregierung mit Landesregierungen anderer Couleur im Bundesrat konfrontiert war. Um ihren Argumenten Nachdruck zu verleihen, reichten die konservativ regierten „Südländer“ 1998 Organklagen vor dem Bundesverfassungsgericht gegen die Reform des Finanzausgleichs zwischen Bund und Ländern ein, dem sie einen Verstoß gegen das Verfassungsgebot eines ,angemessenen Ausgleichs" bescheinigten (Große Hüttmann 1999: 112). Die Parteien und Länder stimmten zunehmend darin überein, dass es zu Änderungen am föderalen System kommen müsse, konnten sich aber nicht auf ein Dezentralisierungsparadigma einigen; die Reform verzögerte sich. Das Urteil des Bundesverfassungsgerichts zur Juniorprofessur im Juli 2004, die als unzulässiger 
Eingriff in die Gestaltungsfreiheit der Länder interpretiert wurde, schwächte die Verhandlungsposition des Bundes, und es kam zu einem gewissen Einlenken. Doch erst im zweiten Anlauf wurde 2006 unter einer nunmehr GroBen Koalition mit der Föderalismusreform eine umfassende, aber in der Gesamtwirkung doch eher bescheidene De-Unitarisierung vorgenommen. Bei aller sichtbaren „Pfadabhängigkeit“ des deutschen Föderalismus sind also doch auch Wandlungstendenzen möglich und erkennbar, wenngleich sie sich typischerweise eher in vielen Einzeländerungen denn in großen Reformen materialisieren (Lehmbruch 2002; Grotz 2009).

\section{Grundrechtsänderungen - hoher Konsensbedarf}

Gemäß Art. 19 Abs. 2 GG darf ein Grundrecht in keinem Falle in seinem Wesensgehalt angetastet werden. Die negativen historischen Erfahrungen mit der Einschränkung bürgerlicher Freiheiten begründeten nicht nur diese Garantie, sondern eine generelle Sensibilität bei Grundrechtsänderungen, die denn auch regelmäßig harte politische Konflikte provozierten. Trafen eine hohe normativ-ideelle Konfliktlast und der Wunsch nach verfassungsrechtlicher Absicherung zusammen, die vor einem Zugriff durch tagespolitische Mehrheiten besser schützt als einfache Gesetze, so mündeten die Verfassungsnovellierungen in ebenso umfassende wie detaillierte Textergänzungen. Dies gilt für vier Fälle: erstens für die 1956 eingefügte „Wehrverfassung“, ${ }^{4}$ zweitens für die 1968 festgeschriebenen Möglichkeiten einer Einschränkung der Grundrechte zum Schutze der freiheitlichen demokratischen Grundordnung oder des Bestandes oder der Sicherung des Bundes oder eines Landes, die Möglichkeiten einer Zwangsverpflichtung von Männern für militärische und zivile Aufgaben im Verteidigungs- oder Spannungsfall sowie von Frauen für zivile Dienstleistungen (Art. 9 Abs. 3, 10, 11 Abs. 2, 12, 12a, 19 Abs. 4 GG), drittens für die Neuregelung des Rechts auf politisches Asyl 1993 (Art. 16, 16a GG) und viertens für die Ermöglichung einer befristeten akustischen Überwachung der Wohnung tatverdächtiger Personen bei schweren Straftaten (Art. 13 Abs. 3-6 GG), die an verschiedene Auflagen gebunden ist. ${ }^{5}$

Die ins Grundgesetz aufgenommene Möglichkeit, Freiheitsrechte der Bürger beschränken zu können, war nicht das vorrangige Ziel der Initiatoren. Vielmehr sollte die Verfassung der Bundesrepublik an veränderte Rahmen-

4 Dies waren Regelungen zum Wehrdienst, zum Wehrersatzdienst aus Gewissensgründen, zum Verbot, Frauen zum Dienst mit der Waffe „zu verwenden“ sowie zur Einschränkung von Grundrechten während des Wehr- oder Ersatzdienstes und durch Gesetze, die der Verteidigung einschließlich des Schutzes der Zivilbevölkerung dienen (Art. 12, 17 GG).

5 Für eine richterliche Anordnung bedarf es konkreter Verdachtsmomente auf eine schwere Straftat, zudem müsste die Erforschung des Sachverhalts bei alternativen Ermittlungsverfahren „,unverhältnismäßig erschwert“ oder „aussichtslos“ sein. Weitere Details beziehen sich etwa auf den Fall von „Gefahr im Verzuge“. 
bedingungen angepasst werden: Wehrverfassung und Notstandsgesetzgebung wurden als wichtige Regularien einer ,normalen“ Demokratie gesehen und von den Alliierten zur Voraussetzung für die Aufhebung wichtiger Vorbehaltsrechte im Bereich der inneren Sicherheit benannt (Glaeßner 1999: 274). Ähnlich wie beim neuen Asylrecht und dem „Großen Lauschangriff“ richtete sich die Kritik vor allem auf die Ausgestaltung der verfassungsrechtlichen Regularien (vgl. Busch 2006: 37, 1999: 557f.), und eben dies spiegelt sich in Verfassungsklauseln in einfachgesetzlichem Stil.

Die weniger umstrittenen grundrechtsrelevanten Änderungen sind hingegen knapp gefasst, so die 1994 erfolgte Ergänzung, dass der Staat die Durchsetzung der Gleichberechtigung von Frauen und Männern fördere und auf die Beseitigung bestehender Nachteile hinwirke sowie dass niemand wegen seiner Behinderung benachteiligt werden dürfe. Ähnlich minimalistisch formuliert war das auf Initiative der FDP Ende 2000 eingefügte Verbot, Frauen zum Dienst an der Waffe zu verpflichten (Art. 12a Abs. 4 GG), mit dem die Parteien auf ein Urteil des Europäischen Gerichtshofs für Menschenrechte reagierten. ${ }^{6}$

Diese Beispiele sollten jedoch nicht zu dem Trugschluss führen, Grundrechteerweiterungen oder zusätzliche Staatszielbestimmungen seien generell weniger umstritten. Von konservativer Seite wurden in den 1990er Jahren beispielsweise alle Vorschläge abgewiesen, ein Recht auf Arbeit, auf Wohnung, auf Bildung, auf Gesundheit, auf soziale Sicherheit, auf Mitmenschlichkeit oder gesicherte Umwelt in die Verfassung aufzunehmen. Eingebracht worden waren diese Ideen nicht nur, aber besonders von seiten der Ostdeutschen, die sie teils auch in ihre Landesverfassungen schrieben. Sie fanden jedoch auf Bundesebene als „utopische“, „einem freiheitlich-demokratischen System wie dem unserer Sozialer Marktwirtschaft wesensgemäß fremde bzw. in ihm von vornherein unerfüllbare“ Forderungen (Scholz 1995: 40) keine verfassungsändernden Mehrheiten. „Diese Ordnung mit der Forderung nach einem auch institutionellen bzw. normierten ,Wertewandel' ${ }^{`} \mathrm{zu}$ konfrontieren, hieße sich auf ein ebenso gefährliches wie letztendlich werte-verneinendes Spiel einzulassen“ (ebd.: 43). Dass Grundrechte- und Staatszieländerungen fast zwangsläufig mit weltanschaulich-religiösen Konflikten verbunden sind und daher entgegen ihren Absichten häufig nicht integrieren, sondern desintegrieren (Preuß 1995: 45), war ein weiterer Einwand gegen sie.

6 Der Gerichtshof, dessen Urteile in der Bundesrepublik einen auf Selbstverpflichtung der Bundesrepublik basierenden einfachgesetzlichen Charakter haben, hatte im selben Jahr der Klage einer Deutschen stattgegeben, der ein Dienstverhältnis mit der Bundeswehr im nichtzivilen Bereich verwehrt worden war. 


\section{Ein semi-souveräner Staat? Grundgesetz und Europäische Union}

Die Einfügung des Europäisierungsgebots lief Anfang der 1990er Jahre der deutsch-deutschen Einigung in der Verfassungsdebatte den Rang ab (Glaeßner 2006: 347; 349). Der „Europa-Artikel“ 23 GG und weitere Änderungen wurden Ende 1992 im Zuge der Schaffung der Europäischen Union als politischer Gemeinschaft mit dem Vertrag von Maastricht angenommen. Dies lag im Interesse der damaligen Bundesregierung unter Helmut Kohl, die Vorbehalte der ehemaligen Siegermächte gegenüber einer deutschen Wiedervereinigung durch eine freiwillige Selbstbindung in die europäische Staatengemeinschaft von vornherein ausräumen wollte. Allerdings knüpft Art. 23 GG wesentliche Integrationsschritte an eine vorherige Zustimmung durch verfassungsändernde Mehrheiten und gibt damit nicht pauschal Souveränität ab. Überdies werden die bundesdeutschen Ordnungsprinzipien Demokratie, Rechtsstaatlichkeit, Sozialprinzip und Föderalismus in Verbindung mit Subsidiarität sowie ein dem Grundgesetz vergleichbarer Grundrechtsschutz explizit als Grundsätze einer EU genannt, an der sich die Bundesrepublik beteiligen darf. Einer möglichen Anpassung an konkurrierende Ordnungsprinzipien wird damit - auch in Reaktion auf das Urteil des Bundesverfassungsgerichts zum Maastricht-Vertrag - vorgebeugt (Glaeßner 2006: 359).

Im Übrigen regelt Art. 23 GG vor allem die Informationspflicht der Bundesregierung gegenüber Bundestag und Bundesrat und deren Mitwirkungsrechte in der Europapolitik. Die Länder hatten die Zustimmung zu den Grundgesetzänderungsvorschlägen der laut Einigungsvertrag gebildeten Gemeinsamen Verfassungskommission davon abhängig gemacht, dass ihnen beziehungsweise dem Bundesrat erhebliche Mitsprache bei der Festlegung und Vertretung der deutschen Position für die Rechtsetzung im Rat der EU eingeräumt wurde (Grimm 2005). Generell achteten die in Bundestag und Bundesrat vertretenen Akteure im Zuge zunehmender Vergemeinschaftung stärker darauf, ihre Mitsprache in EU-Angelegenheiten möglichst umfassend zu sichern. In diese Richtung weist auch die am 8. Oktober 2008 ausgefertigte, aber noch nicht in Kraft gesetzte Änderung des Grundgesetzes in Art. 23, 45 und 93 GG, die die Abgabe weiterer Kompetenzen an die EU ermöglicht, jedoch gleichzeitig Bundestag und Bundesrat das Recht zuschreibt, wegen Verstoßes eines Gesetzgebungsaktes der EU gegen das Subsidiaritätsprinzip vor dem Europäischen Gerichtshof zu klagen. Auf Antrag eines Viertels seiner Mitglieder ist der Bundestag dazu verpflichtet (BGBl. 2008 I: 1926).

Angesichts einer fehlenden europäischen Verfassung wurde der Text des Grundgesetzes bislang nur soweit „europäisiert“, wie sich dafür in den politischen Vertretungsorganen eine breite Mehrheit fand. Auch wenn ganz unübersehbar Kompetenzen abgetreten wurden, liegt die Verfassungssouveränität weiter in nationaler Hand.

Insgesamt waren für die Bundesrepublik jeweils nicht sehr weitreichende Änderungen der Staatsorganisation die Regel, größere Reformen wie 1968/69, 
1994 oder 2006 (Grotz 2009) oder normativ-ideell ausgerichtete Modifikationen die Ausnahme. Die umfassenden Neuerungsvorschläge der vom 6. Bundestag 1971 eingesetzten Enquête-Kommission „Verfassungsreform“ und der infolge des Einigungsvertrages 1991 eingesetzten Gemeinsamen Verfassungskommission von Bundestag und Bundesrat blieben weitgehend folgenlos, weil es an einem verfassungsänderndem Konsens über die Ausgestaltung fehlte (Glaeßner 1999: 193ff.; Batt 1996). Ähnliches war der eingesetzten Bundesstaatskommission widerfahren (A. Benz 2005), bevor sich die Große Koalition 2006 entschied zu handeln. In der Summe seiner Änderungen wandelte sich das Grundgesetz gegenüber 1949 durchaus erheblich (wenn man allein schon die Gebietserweiterungen, die Wiederbewaffnung und die Möglichkeit von Auslandseinsätzen der Bundeswehr betrachtet), doch standen die zentralen politischen Ordnungsprinzipien nicht zur Disposition.

\section{Impliziter Wandel des Grundgesetzes: konkretisierend, selten normschöpfend}

Die Vorstellung sei „falsch, das Grundgesetz bestehe nur aus seinem Text“, erklärte unlängst Andreas Voßkuhle, Richter am Bundesverfassungsgericht. „Es besteht auch aus weit über hundert Bänden Bundesverfassungsgerichtsentscheidungen, die diesen Text konkretisieren“" (FAZ 01.05.2008). Das Grundgesetz gilt ,praktisch so, wie das Bundesverfassungsgericht es auslegt“ (Smend 1963, zit. in: Schuppert 1980: 1). Diese Rolle eines notfalls auch die Politik beschränkenden Organs hatten die Verfassungsväter und -mütter dem Gericht nicht zugedacht oder zumindest unterschätzt, wie überraschte Reaktionen Konrad Adenauers und anderer hochrangiger Politiker auf Urteile des Gerichts in der frühen Bundesrepublik und die nachfolgenden Kompetenzkonflikte zeigten (Wesel 2004: 76ff.).

Das Bundesverfassungsgericht definierte 1952 in einem Urteil zur Sozialistischen Reichspartei (SRP) die „freiheitliche demokratische Grundordnung" als so genannten Kernbereich des Grundgesetzes, der unter besonderen Schutz zu stellen und gegenüber anderen Normen stärker zu gewichten sei. Den in Art. 18, 21 Abs. 2 und 91 Abs. 1 GG bereits enthaltenen Begriff interpretierte sie als eine Ordnung, die unter Ausschluss jeglicher Gewalt- und Willkürherrschaft eine rechtsstaatliche Herrschaftsordnung auf der Grundlage der Selbstbestimmung des Volkes nach dem Willen der jeweiligen Mehrheit und der Freiheit und Gleichheit darstellt. $\mathrm{Zu}$ ihren grundlegenden Prinzipien zählen, so das Gericht, die Achtung vor den im Grundgesetz konkretisierten Menschenrechten, die Volkssouveränität, die Gewaltenteilung, die Verantwortlichkeit der Regierung, die Gesetzmäßigkeit der Verwaltung, die Unabhängigkeit der Gerichte, das Mehrparteienprinzip und die Chancengleichheit für alle politischen Parteien mit dem Recht auf verfassungsmäßige Bildung 
und Ausübung der Opposition (Wesel 2004: 85). Diese Definition wurde wegweisend für die entsprechende Gesetzgebung der Bundesrepublik und diente als implizite Referenz für mehrere Verfassungsänderungen (1968: Art. 10 Abs. 2, 11 Abs. 2, 87a Abs. 4 GG; 1972: Art. 73 Nr. 10 GG).

In jeder Konkretisierung von Verfassungsnormen materialisiert sich Wandel. Der Sinn des ursprünglichen Verfassungstextes wird verengt, verschoben, ausgeweitet oder gewichtet. Ohne eine solche Textauslegung ist eine auf spezifische Klagefälle bezogene (Verfassungs-)Rechtsprechung gar nicht möglich. Beispielsweise diente die Definition der freiheitlichen demokratischen Grundordnung dem Zweck, die Verfassungsmäßigkeit der SRPAktivitäten intersubjektiv nachprüfbar „,messen“ und ein Verbot begründen zu können (Wesel 2004: 85). ${ }^{7}$ Ein zu weit gefasstes Verständnis impliziten Verfassungswandels ${ }^{8}$ würde insofern die auf ihm beruhenden Bewertungen banalisieren. Für die Fragestellung dieses Aufsatzes, wie sehr sich die deutsche Verfassung gegenüber 1949 verändert hat, sind daher im Folgenden nur jene Entscheidungen des Bundesverfassungsgerichtes relevant, die das vorhandene konstitutionelle Normprogramm erweiterten beziehungsweise die Normadressaten mit neuen Verhaltensanforderungen konfrontierten (Kneip 2009; Bryde 1982: 21f.). Dass die Bewertung nicht immer zweifelsfrei erfolgen kann, provozierte eine umfassende Literatur zum Verhältnis zwischen Politik und Recht (Reutter 1998).

Im All-Demokratien-Vergleich wird das Bundesverfassungsgericht meist zu den mächtigsten Verfassungsgerichten gezählt, weil es ausgedehnte Kompetenzen besitzt, die es selbst noch verfeinerte, so in Form einer Teilanullierung von Gesetzen (Vanberg 2005: 92, Fn. 36; Piazolo 1995: 7). Allerdings verfügt es über keine Sanktionsmittel, um die Durchsetzung seiner Entscheidungen zu gewährleisten (Isensee 1995a: 53). Im nicht nur kurzfristigen, mit einem Urteil endenden, sondern langfristigen Verhältnis zur Politik sind seine stärksten „Waffen“ Reputation und Öffentlichkeit beziehungsweise Transparenz (Vanberg 2005). Das Bundesverfassungsgericht kann außerdem allenfalls dort übermächtig sein, wo das Grundgesetz keine konkreten Regelungen vorgibt. Denn das Normschöpfungspotenzial der Gerichtsbarkeit steigt, ceteris paribus, je weniger inhaltlich bestimmt die Verfassungsnormen (Böckenförde 2000: 153), je geringer also die Regelungsdichte der Verfassung oder einer Norm. Nimmt man die Regelungsdichte der Gesamtverfassung zum Maßstab, liegt das Bundesverfassungsgericht hinsichtlich seiner Möglichkeiten im unteren Mittelfeld der Demokratien. ${ }^{9}$ Aber die Regelungsdichte va-

7 Für eine Kritik des Urteils vgl. Wesel 2004.

8 Verfassungswandel wird hier in einem politikwissenschaftlichen Verständnis äquivalent zum Terminus „constitutional change“ verwendet. Zur abweichenden Diskussion um den Begriff im deutschen Staatsrecht siehe unter anderen Böckenförde 1998 und Voßkuhle 2004.

9 Grundlage der Bewertung ist ein Kombinationsmaß, das die Länge der Verfassung in Zeilen sowie die Anzahl der Artikel berücksichtigt; vgl. Lorenz 2008: 385. 
riiert je nach Artikel. Wo ein besonderes Interesse oder eine spezifische Konfliktlast zu differenzierten Normen führte, etwa beim Europa-Artikel, beim „Großen Lauschangriff“ oder den enumerativ gehaltenen Gesetzgebungskompetenzen, sind die Spielräume der Rechtsprechung begrenzt.

Die zentrale institutionelle Voraussetzung für eine Aktivierung der verfassungsgerichtlichen Kompetenzen besteht darin, dass überhaupt geklagt wird und das Gericht sich des Vorgangs annimmt. Dies kann je nach Art des Verfahrens durch einfache Bürger, gesellschaftlich organisierte Gruppen, untergeordnete Gerichte oder politische Akteure geschehen. Die „,̈̈berwältigende Mehrzahl der Klagen und Verfahrensinitiativen“" vor dem Bundesverfassungsgericht ging auf dem Weg der Verfassungsbeschwerde von einzelnen Bürgern aus (Stüwe 1997: 287), der „Krönung des Rechtsstaates“ (Clemens 1995: 14). Über 90 Prozent dieser Beschwerden, auf deren Möglichkeit die besondere Reputation des Gerichts nicht unerheblich gründet, scheiterten schon in der Vorprüfung, wurden also noch nicht einmal angenommen. In den ersten 50 Jahren seit Bestehen des Bundesverfassungsgerichts hatten von den fast 130.000 Verfassungsbeschwerden weniger als 2,5 Prozent Erfolg (Wesel 2004: 8).

Trotz ihres geringen Anteils waren die verfassungsgerichtlichen Verfahren, die von der Opposition beantragt wurden, oft aufsehenerregend und gesellschaftlich brisant (Stüwe 1997). Nicht selten betrafen solche Normenkontrollverfahren konfliktträchtige Materien. Grundsätzlich traten die Kläger Kosten-Nutzen-kalkulierend auf: Sie konzentrierten sich auf umstrittene Regelungen, aussichtsreiche Klagen und Fragen, in denen die Aushandlungsoptionen der normalen Gesetzgebung ausgeschöpft schienen (Hönnige 2007; Stone Sweet 2000; Vanberg 2005). Die Klage erfolgte demnach nicht quasistandardisiert.

Wie bei den expliziten Verfassungsänderungen richteten sich die Aktivitäten der Opposition im Hinblick auf das Bundesverfassungsgericht auf verschiedene Politikbereiche und Rechtsgebiete. Sie lassen jedoch auch Schwerpunkte erkennen, die ganz klar dem Postulat rational-sozialen Handelns entsprechen: Die Parteienfinanzierung war am häufigsten und regelmäßig Gegenstand von Verfahrensinitiativen, die von der parlamentarischen Opposition und Landesregierungen ausgingen, die parteipolitisch anders zusammengesetzt waren als die jeweilige Bundesregierung. Hier zeigt sich ein deutlicher Kontrast zum expliziten Verfassungswandel, denn die Parteienfinanzierung war nur einmal - 1983 - Gegenstand einer Grundgesetzänderung. Am zweithäufigsten klagte die Opposition mit dem Ziel, die eigenen Handlungsmöglichkeiten im Parlament zu verbessern. Hierauf richteten sich Verfahren, die von der SPD, den Grünen und der PDS angestrengt worden waren. Den dritten Schwerpunkt bildeten die Außen-, Verteidigungs- und Deutschlandpolitik. Hier war der Bezug zu politischen Vorhaben der Bundesregierungen am konkretesten, die Kläger waren intrinsisch-programmatisch motiviert und/oder darauf bedacht, mit der symbolischen Klage vor dem Gericht die 
Aufmerksamkeit und Stimmen der Wähler zu maximieren. Dies gilt etwa für die Grünen und die Linke, für die Friedenspolitik traditionell eine große Bedeutung besitzt. Geklagt wurde Anfang der 1950er Jahre, 1973, in den 1980er und 1990er Jahren (Stüwe 1997: 211ff.). Ebenfalls regelmäßig hatte das Gericht über das Verhältnis von Bund und Ländern zu urteilen, besonders über die föderale Finanzverteilung (Korioth 2006).

Hervorzuheben ist, dass die Kläger zwar immer versuchten, in einem „Spiel über Bande“ eigene Interessen durchzusetzen, etwa die Anullierung kritisierter Gesetze, dass jedoch in den meisten Verfahren vor dem Bundesverfassungsgericht weder die Kläger noch das Bundesverfassungsgericht einen Wandel der Verfassung selbst intendierten oder er durch Entscheidung herbeigeführt wurde (Kneip 2009). ${ }^{10}$ Dies fällt besonders auf, weil Initiativen für formelle Grundgesetzänderungen ja sehr häufig eingebracht wurden. Offensichtlich wird das Bundesverfassungsgericht als letzte Option der Nutzenmaximierung innerhalb der konstitutionellen Spielregeln wahrgenommen, aber nicht der Nutzenmaximierung über veränderte Spielregeln. Entsprechend dem Rationalitätspostulat ist dies dahingehend zu deuten, dass die politischen Akteure ein Konsens eint, sich diese Option nicht vom Bundesverfassungsgericht nehmen zu lassen, und das Bundesverfassungsgericht selbst bestrebt ist, die Achtung der eigenen Urteile trotz künftiger Machtwechsel zu sichern, also nicht einseitig Partei zu ergreifen (Vanberg 2005).

Grundsätzlich urteilten die Richter eher strukturkonservierend, unabhängig von ihren politischen Affinitäten (Hönnige 2007: 243). Das Gericht war nicht immun gegenüber politischen Stimmungslagen, verhielt sich aber eher zurückhaltend als offensiv (Vanberg 2005). Bei Konflikten, für deren Klärung das Bundesverfassungsgericht ursprünglich zuvorderst geschaffen worden war, nämlich zwischen und in den Verfassungsorganen, sowie bei Klagen, bei denen die Absicht der Politik, das Gericht zu instrumentalisieren, offensichtlich war, hielt es sich besonders zurück, indem es beispielsweise auf die Geschäftsordnungsautonomie des Bundestages verwies. Insgesamt scheiterten die Klagen der Opposition durchaus oft (Stüwe 1997: 283; Reutter 2001). Zurückhaltung zeigte das Gericht auch bei mehreren Versuchen, formelle Grundgesetzänderungen mit Hilfe einer Verfassungsklage nachträglich zu verhindern. Dies gilt für das Abhörurteil (BVerfGE 30,1), die Urteile zur Bodenreform I (BVerfGE 84, 90; 94, 12), zum Vertrag von Maastricht beziehungsweise dem Europa-Artikel (BVerfGE 89, 155) und zur Asylnovelle (BVerfGE 94, 094) (Kneip 2009).

Föderative Konflikte spielen in der Verfahrensstatistik eine geringe Rolle. Das Gericht hatte bereits seit seiner Einrichtung 1951 ,nicht vorrangig klassische Fragen der sachbereichsbezogenen vertikalen Kompetenzabgrenzung“ zu

10 Grundlage der Bewertung ist die oben erwähnte Definition von Verfassungswandel. Eine „normale“ Interpretation wird hier nicht als Verfassungswandel eingestuft, weil das, wie erwähnt die Aussagen banalisieren würde. 
entscheiden, sondern „Konflikte im kooperativen und solidarischen Miteinander von Bund und Ländern, eingeschlossen Versuche der Länder, auf die Bundespolitik einzuwirken, oder umgekehrt des Bundes, seine Vorstellungen auch in den Ländern zur Geltung zu bringen“ (Korioth 2006: 393). Es stärkte zwar die Autonomie der Länder, betonte aber auch die Ländergleichheit und den Bedarf solidarischen Ausgleichs. Dem Trend der politischen Mehrheiten auf Bundesebene folgend, trug es zunächst zum schleichenden Ausbau der Bundesratskompetenzen in der Gesetzgebung bei. Seit der Reform der Finanzverfassung erhielt die Rechtsprechung hier einen neuen Schwerpunkt und bestätigte darin die ,föderativen Grundtendenzen zur Vereinheitlichung der Lebensverhältnisse, zur Unitarisierung, zur breiten Streuung der föderalen Entscheidungsfindung und zur Verantwortungsteilung“ (ebd.: 402). Erst seit Beginn der 1990er Jahre stärkte die Verfassungsrechtsprechung eine leichte Reföderalisierung auch hier aber gemäß dem allgemeinen, oben geschilderten Trend der expliziten Verfassungsentwicklung (ebd.: 402ff.).

Größere Effekte als auf das Kräfteverhältnis zwischen den politischen Akteuren hatte die Rechtsprechung des Bundesverfassungsgerichts in Bezug auf die Grundrechte, denen die Richter 80 bis 90 Prozent ihrer Zeit widmeten (Wesel 2004: 360). Die meisten seiner konstitutionellen Schlüsselentscheidungen, das heißt Entscheidungen, die bestehende Verfassungsnormen erkennbar erweiterten und als Maßstäbe der weiteren Rechtsauslegung dienten, betrafen denn auch die Grundrechte, wobei auch sie häufiger einen nichtintendierten als einen intendierten Verfassungswandel beinhalteten (Tabelle 2; Kneip 2009).

Tabelle 2: $\quad$ Schlüsselentscheidungen des Bundesverfassungsgerichts mit verfassungswandelndem Charakter

\begin{tabular}{|l|l|}
\hline Intendierter Verfassungswandel & Nicht-intendierter Verfassungswandel \\
\hline Erste Rundfunkentscheidung (Deutschlandfern- & Elfes (BVerfGE 6, 32) \\
sehen) (BVerfGE 12, 205) & Lüth (BVerfGE 7, 198) \\
Schwangerschaftsabbruch I (BVerfGE 39, 1) & Apothekenurteil (BVerfGE 7, 377) \\
Heilpraktiker (BVerfGE 78, 179) & Aktion Rumpelkammer (BVerfGE 24, 236) \\
Bundeswehreinsatz Somalia/AWACS (BVerfGE & Mephisto (BVerfGE 30, 173) \\
$90,286)$ & Strafgefangene (BVerfGE 33, 1) \\
EG-Fernsehrichtlinie (BVerfGE 92, 203)* & Numerus Clausus I (BVerfGE 33, 303) \\
& Abgeordnetendiäten (BVerfGE 40, 296) \\
& Volkszählung (BVerfGE 65, 1) \\
& Parteienfinanzierung II (BVerfGE 85, 264) \\
& Transsexuelle II (BVerfGE 88, 087) \\
& Vermögensteuer (BVerfGE 93, 121) \\
\hline
\end{tabular}

* Durch eine formelle Verfassungsänderung wurden die Inhalte des Urteils bereits vorweggenommen

Quelle: Kneip 2009. 
$\mathrm{Zu}$ den verfassungswandelnden Entscheidungen zählten beispielsweise das Urteil zur „Aktion Rumpelkammer“, in dem das Bundesverfassungsgericht das Grundrecht auf Religionsfreiheit erstmals umfassend ausarbeitete, oder das Urteil zur Volkszählung 1983, in dem es das so genannte „Recht auf informationelle Selbstbestimmung" schuf. Auch die Schaffung des neuen Grundrechts ,auf Gewährleistung der Vertraulichkeit und Integrität informationstechnischer Systeme" durch das Gericht im Urteil zu Onlineuntersuchungen vom 27. Februar 2008 kann als Meilenstein des Verfassungswandels bezeichnet werden, obgleich das junge Datum des Urteils es noch nicht zulässt, es als Schlüsselentscheidung gemäß den obigen Kriterien zu klassifizieren. Ähnlich wie beim Recht auf informationelle Selbstbestimmung leitete das Gericht dieses Recht aus dem allgemeinen Persönlichkeitsrecht ab (Art. 2 Abs. 1 i.V.m. Art. 1 Abs. 1 GG) (BVerfG, 1 BvR 370/07).

Hatte das Grundgesetz 1949 nur Abwehrrechte des Bürgers gegen den Staat enthalten, so schuf der erste Senat des Bundesverfassungsgerichts inzwischen ein ganzes „Wertesystem“, „das in alle Bereiche des Rechts übergreift (...) und an dem sich sogar Schutzpflichten des Staates ergeben, der deshalb Gesetze mit bestimmten Inhalten zum Schutz einzelner Grundrechte erlassen muss", etwa hinsichtlich der Urteile zum Schwangerschaftsabbruch (Wesel 2004: 362). Mit dem frühen „Lüth-Urteil“ verankerte es zudem die fundamentale Drittwirkung der Grundrechte in der bundesdeutschen Rechtsordnung. Die Stärke ihrer Wertgebundenheit und ihrer Verzahnung von Verfassungsrecht und einfachem Recht sind seither ein „Alleinstellungsmerkmal" des deutschen Rechts (Henne 2006: 142). Das Grundgesetz wurde damit zu einer Art politisches Programm, das der Staat auszuführen hat.

Während das Gericht hinsichtlich der Grundrechte das Verfassungsrecht und die verfassungsrechtliche Überprüfung weiter und intensiver in das ,einfache Recht" hinein erstreckte, legte es gleichzeitig (mit Unterstützung der Politik qua Gesetz) die Zugangshürde für die Verfassungsbeschwerdeführer höher, um eine intensive Einzelfallprüfung überhaupt zu ermöglichen. Gegenüber der Politik beließ es mehr Entscheidungsfreiräume als gegenüber den Fachgerichten und Verwaltungsbehörden (Clemens 1995: 20ff., 25), was angesichts der institutionellen Rahmenbedingungen nur rational erscheint. Es baute da seinen Einfluss aus, wo weniger Widerstände und Risiken für die Akzeptanz eigener künftiger Entscheidungen durch andere bestehen, und hielt sich gerade bei der Aufgabe zurück, für die er geschaffen worden war: der Schlichtung von Streit zwischen den Verfassungsorganen.

Das Bundesverfassungsgericht ging aber durchaus auch weiter als die Politik, besonders hinsichtlich der Gleichberechtigung. ${ }^{11}$ Bedeutungsvoll war

11 Bereits in den 1950er Jahren erklärte es häufig Gesetzesregelungen für verfassungswidrig, die der im Grundgesetz festgeschriebenen Norm der Gleichberechtigung von Mann und Frau widersprachen. Dass sich dafür gerichtsintern besonders Richterinnen einsetzten (HohmannDennhardt 2006; Wesel 2004), zeugt davon, dass die Urteile nicht von „,neutralen“, interessenenthobenen Personen formuliert werden (Limbach 1999: 142; Hönnige 2007). 
und bleibt angesichts der Rahmenbedingungen vermutlich auch in Zukunft die Rechtsprechung zur staatlichen Abwehr von Terrorismus und organisierter Kriminalität sowie zum Datenschutz. Die jüngeren Verfahren zum Luftsicherheitsgesetz, zur Onlinedurchsuchung, zu Autokennzeichenscans und zur Vorratsdatenspeicherung belegen, dass die Sicherheit der Allgemeinheit und die Freiheit des Einzelnen angesichts veränderter politischer Rahmenbedingungen nur unter Schwierigkeiten neu austariert werden können (Glaeßner 2003). Die Problematik ist gleichzeitig für alle Beteiligten besonders wichtig, weshalb der Gang nach Karlsruhe nicht gescheut wird. ${ }^{12}$ In der Spruchpraxis war die Tendenz erkennbar, dem Staat den Eingriff in die Grundrechte der Bürger grundsätzlich zu erlauben, um sich und sie zu schützen, ihn jedoch an strengere Bedingungen zu knüpfen, als der Gesetzgeber vorsah (Biermann 2008a, 2008b). Der Politik wurden hier mit Verweis auf einzelne, höher gewichtete Verfassungsnormen zum Schutze der Privatsphäre Grenzen aufgezeigt.

In einem anderen Punkt, der für die Souveränität der Bundesrepublik von essentieller Relevanz ist, hält sich das Bundesverfassungsgericht zurück: hinsichtlich der EU-Integration. Die (im Zeitverlauf nicht konsistente) Urteilspraxis verharrt im konventionellen Dualismus von Staats- und Völkerrecht. Das Gericht deklarierte zwar einen grundsätzlichen Vorrang des Gemeinschaftsrechts, aber nur solange keine Entstaatlichung stattfindet und soweit Letzteres nicht aus dem Kern der Verfassung ausbricht. In seinem Maastricht-Urteil konzedierte es eine Integrationsoffenheit des Grundgesetzes, bezeichnete die EU aber als „Staatenverbund“, der „,von den Mitgliedstaaten getragen wird und deren nationale Identität achtet" (BVerfGE 89, 155 [183, 181]). Entsprechend ist beispielsweise die Unions- aus der Staatsangehörigkeit abgeleitet. Der Vorbehalt der nationalen Souveränität wurde somit beibehalten. Das Bundesverfassungsgericht übt seine Gerichtsbarkeit über die Anwendbarkeit von abgeleitetem Gemeinschaftsrecht in Deutschland in einem „Kooperationsverhältnis“ zum Europäischen Gerichtshof aus, „indem der EuGH den Grundrechtsschutz in jedem Einzelfall für das gesamte Gebiet garantiere, das Bundesverfassungsgericht sich deshalb auf eine generelle Gewährleistung der unabdingbaren Grundrechtsstandards (...) beschränken könne“ (Kruis 1995: 131; ähnlich Mayer 2009; Glaeßner 2006: 350ff.).

Da völkerrechtliche Vereinbarungen keiner Legitimation durch die Bürgerinnen und Bürger bedürfen, trägt das Bundesverfassungsgerichts nicht da$\mathrm{zu}$ bei, die Legitimationsdefizite mancher EU-Politiken aufzuzeigen und $\mathrm{zu}$ beseitigen (van Ooyen 2008: 53). Der ,(...) jenseits der eingerichteten europäischen Institutionen viel zu schwach entwickelte europäische politische Prozess pluralistischer Gruppen - der wohl das Ergebnis einer seit Jahrzehnten betriebenen etatistischen Integration ist - reduziert sich aus der bundes-

12 Dies schließt nicht aus, dass es auch Bürgerrechtsaktivisten gibt, die sich besonders engagierten, wie Gerhart Baum oder Burkhard Hirsch. 
verfassungsgerichtlichen Sicht einer ,staatlich vermittelten Volksdemokratie“ bloßer ,Legitimationsketten“ allenfalls auf ein Randproblem“ (van Ooyen 2008: 58). Diese Praxis konfligiert mit der sonstigen Sorge des Gerichts um den Grundrechtsschutz. So sah das Bundesverfassungsgericht in seinem Urteil zum Europäischen Haftbefehlsgesetz des Bundestags 2004 im geringen Einfluss des Europaparlaments und des Bundestags auf den zugrunde liegenden Rahmenbeschluss des Rats zum EU-Haftbefehl 2002 kein Problem. Das Gesetz ermöglicht bei 32 aufgelisteten Straftaten, darunter Terrorismus, die Auslieferung ohne Überprüfung des Vorliegens der beiderseitigen Strafbarkeit und damit die Absenkung des Rechtsschutzes auf das Maß des ,punitivsten" EU-Staates (van Ooyen 2008: 52).

Durch das vorläufige Scheitern der Annahme einer EU-Verfassung steht eine abschließende Klärung des Verhältnisses zwischen nationalen Verfassungsregelungen und supranationalem Recht weiter aus, aber die Verordnungen und Richtlinien, die der Europäische Gerichtshof auslegt, wachsen weiter. Angesichts aktiver Integrationsgegner wird das Bundesverfassungsgericht nicht umhin kommen, zumindest in Teilfragen immer wieder Position im Kompetenzstreit zu beziehen, wie aktuell betreffend den Vertrag von Lissabon oder die EU-Regelungen gegen Altersdiskriminierung.

\section{Resümee: Umfassender Wandel, Ordnung im Kern}

Retrospektiv wurde die Stabilität der bundesdeutschen Demokratie in institutionalistischer Deutung besonders auf die im Grundgesetz gezogenen „Lehren aus Weimar“ zurückgeführt. Allerdings lässt sich ein unmittelbarer $\mathrm{Zu}$ sammenhang ,zwischen der Etablierung der NS-Diktatur und den strukturellen Defizienzien des Weimarer politischen Systems und namentlich der Reichsverfassung“ nicht nachweisen (Mommsen 1998: 2). Auch scheint fraglich, ob das Grundgesetz allein stabilisierend gewirkt hätte. Ohne das Europäische Wiederaufbauprogramm, das auf dem Marshall-Plan basierte und wenige Monate nach der Verabschiedung des Grundgesetzes umgesetzt wurde, ohne das Wirtschaftswunder und die geopolitischen Rahmenbedingungen wäre die Erfolgsgeschichte der Verfassung kaum zu erklären. Zwar boten „die verfassungsrechtlichen Regelungen und das institutionelle Gefüge der Bundesrepublik eine solide Basis für eine Konsolidierung der Demokratie“, doch stand diese ,in den Anfangsjahren auf äußerst schwachen Füßen“. Es waren, wie Glaeßner (1999: 46f.) schreibt, ,vor allem die außerordentlich günstigen wirtschaftlichen und sozialen Rahmenbedingungen der 50er und frühen 60er Jahre, die dazu beitrugen, daß sich die ordnungspolitischen Entscheidungen der Jahre 1948/49 als tragfähige Grundlage für den zweiten Versuch erwiesen, in einem Teil Deutschlands eine funktionierende demokratische Ordnung zu etablieren“. 
Die verfassungs- und institutionenpolitischen Entscheidungen und Weichenstellungen von 1949 bilden in dieser Argumentation, die empirisch durch die weltweiten Systemwechselprozesse der letzten Jahrzehnte bestätigt wurde, ein wesentliches Element der Stabilität und Kontinuität der zweiten deutschen Demokratie, dürfen aber nicht losgelöst von den Rahmenbedingungen ihres Wirkens betrachtet werden. Die „schönste“ Verfassung garantiert allein keine gut funktionierende Demokratie, umgekehrt kann eine weniger ,schöne" Verfassung unter bestimmten Rahmenbedingungen einen wichtigen Beitrag zur Stabilisierung des Systems leisten (Merkel et al. 1996; Bos 2004; Lorenz 2001). Der Kontext beeinflusst die Akteure und ihre Machtkonstellationen sowie dadurch die Verfassungswirklichkeit. In dieser Hinsicht hatte die Bundesrepublik Glück.

Inzwischen sind die „Geburtsfehler“ des Grundgesetzes weithin vergessen. Die Akzeptanz und Folgebereitschaft sowohl bei den politischen Akteuren als auch bei den Bürgern ist enorm. In der alten Bundesrepublik ersetzte es vielfach den Bezug auf die Nation im Sinne eines Verfassungspatriotismus (Sternberger 1982); diese Idee wird seit einiger Zeit unter anderen Vorzeichen wieder aufgegriffen (unter anderen Leutheusser-Schnarrenberger 2006). Die hohe Akzeptanz geht mit einem vielfach geringen Wissen über die Verfassungsinhalte und die Charakteristika der bundesdeutschen Verfassungspolitik einher; das Grundgesetz genießt insofern oft einen unspezifischen Vertrauensvorschuss. Dies schloss, wie zu sehen war, Änderungen nicht aus.

Tatsächlich ist das Grundgesetz, ,heute (...) nicht mehr das von 1949. Die Substanz ist im Wesentlichen dieselbe geblieben, aber vieles ergänzt oder geändert worden, meistens durch Bundestag und Bundesrat" (Wesel 2004: 361). Von den Streitpunkten, die schon während der Verfassungsgebung bestanden, blieben diejenigen - und zwar bis heute - bestehen, die konkrete, starke Interessen politischer Akteure berührten, zuvorderst die Verteilung von Gesetzgebungskompetenzen und Finanzen. Hier konnte die Verfassung nur bedingt einhegen und bändigen. So wurde derjenige der beiden Kernartikel der Finanzverfassung, um den 1948/49 so gerungen wurde und der die Verteilung des Steueraufkommens zwischen Bund und Ländern, seit 1997 auch den Kommunen, regelt (Art. 106 GG), seit der Verabschiedung des Grundgesetzes bereits sechsmal geändert. Diskussionen zu anderen Materien, so zur Stellung des Bundespräsidenten, werden hingegen nur ab und zu aufgewärmt. Insgesamt beschäftigen sich die politischen Akteure in den Grundgesetzänderungen besonders oft mit sich selbst beziehungsweise ihrem internen Kräfteverhältnis im Rahmen des kooperativen Föderalismus, und diese Fragen werden auch regelmäßig an das Bundesverfassungsgericht herangetragen.

Die zentralen Ordnungsprinzipien des Grundgesetzes wurden im Kern nicht angetastet und auch nicht in Form von Verfassungsänderungsinitiativen gehäuft infrage gestellt. Dies sind die ,freiheitliche demokratische Grundordnung", der Föderalismus und die Sozialstaatlichkeit als grundsätzliche Ordnungskonzepte. Das Europäisierungsgebot kam hinzu, steht aber unter natio- 
nalem Vorbehalt. Einige deutliche Grundrechtsänderungen wurden aufgrund intensiver politischer Auseinandersetzungen in detaillierte Regelungen gekleidet. Diese Stabilität im institutionellen Kern der Verfassung zeugt davon, dass es einen normativen Grundkonsens im Großen gibt - durchaus auch im Sinne normativer Pfadabhängigkeit, ${ }^{13}$ wenn man der Ansicht folgt, dass keine Demokratieform gegenüber anderen universell überlegen ist. Sie ist allerdings auch dadurch zu begründen, dass die alltagsaffinen Materien die Akteure eben stärker beschäftigen als die „großen Fragen“, auf die sie oft keine (zumal konsensfähigen) Antworten haben. Ihre Aufmerksamkeit ist dadurch gebunden.

Die Opposition, die mehr in die Kompromissfindung einbezogen wurde, als für die Durchsetzung von Entscheidungen nötig, ging in ihr wichtigen Materien durchaus auch vor das Bundesverfassungsgericht. Sie strebte mit Klagen allerdings keine Änderung von Verfassungsnormen an, sondern eine den eigenen Interessen dienliche Auslegung der Verfassungsnormen. Das Gericht selbst mied aus Opportunitätserwägungen heraus eine einseitige politische Parteinahme, urteilte im Hinblick auf die Verfassung insgesamt eher strukturkonservierend und interpretativ, aber selten normschöpfend. Hinsichtlich der Grundrechte zeigte sich, dass es Verfassungswandel vorantreiben kann; es ist aber nicht darauf bedacht, diese Rolle auszubauen. Während in Bezug auf die Auslegung und Fortbildung des Verfassungsrechts im ,einfachen Recht“ innerhalb des bestehenden Normprogramms durchaus schwer auszumachen ist, wer ,die Fäden in der Hand" halte, der Politiker oder der Jurist (Isensee 1995b: 32; Clemens 1995: 19), kann der qualitative Verfassungswandel demnach doch als Ergebnis hauptsächlich von Politik verstanden werden.

Die Behauptung, ,governing with judges" heiße ,governing like judges“ (Stone Sweet 2000: 1), ist demnach für Deutschland überspitzt. Die Politik verfügt durchaus über Möglichkeiten (sofern sie sich denn einig wird), den institutionellen Bezugsrahmen der Urteile, nämlich das Grundgesetz, zu ändern, um veränderte Rahmenbedingungen und Interessen zu verarbeiten oder sich der Urteile des Bundesverfassungsgerichts $\mathrm{zu}$ entziehen. ${ }^{14}$ Wohl aber erwartet sie ihrerseits vom Gericht Steuerungsleistungen in Fragen, hinsichtlich derer sie noch keinen Konsens gefunden hat. Hintergrund sind oft eine gravierende politische Uneinigkeit und „tiefe Risse im kollektiven Rechtsbewußtsein“, wie im Bereich der Bioethik, Gentechnik, religiösen Bekenntnisse (Guggenberger/Würtenberger 1998: 3; Schuppert/Bumke 2000). Die notwendige Regelung vieler Materien, die die Entwicklung der gesellschaftlichen Realität mit sich brachte, die der Wortlaut Grundgesetz aber infolge mangelnder Einigkeit nicht widerspiegelt, wurde so dem Bundesverfassungsge-

13 Zum Konzept der „Pfadabhängigkeit“vgl. Ackermann 2001.

14 Auch die Antizipation von Gerichtsurteilen, etwa bei Klageandrohung durch politische Mitspieler, ist eine mögliche Form judizialisierter Politik; da diese informelle Beeinflussung aber schwer systematisch zu „messen“ ist, bleibt sie aus der Betrachtung ausgeschlossen; vgl. auch Vanberg 2005: 6f. 
richt überlassen. Hier handelt es sich um eine vorübergehende oder partielle Judizialisierung im Sinne einer Verlagerung von Entscheidungen an das Gericht (Landfried 1984: 10; 1994). Sie greift solange, bis die eigentlichen verfassungspolitischen Gestalter sich einigen können und dabei Normierungsideen des Gerichts aufnehmen, ${ }^{15}$ das insofern die Funktionen eines Normenprüfers und Mediators übernimmt.

Dass dem Bundesverfassungsgericht ungeachtet seiner insgesamt moderaten Rolle Ende der 1970er und Mitte der 1990er Jahre (anlässlich mehrerer umstrittener Urteile) vorgeworfen wurde, es mische sich zu sehr in die Politik ein (Wahl 1998; Limbach 1999: 127f.; 167ff.; Höffe 1999), zeigt, dass die Legitimation von Verfassungswandel zumindest bei als wichtig erachteten Fragen argwöhnisch hinterfragt wird. Dies ist aus demokratietheoretischer Sicht auch wünschenswert. Hier sei nicht nur an die Richterrekrutierung ohne Wahl (und Abwahl) erinnert, sondern auch daran, dass verfassungsrechtliche Diskurse „im Prinzip nur Normen aus Normen ableiten“ können, weil ihnen die ,theoretischen und methodischen Instrumente für die Realanalyse der regelungsbedürftigen gesellschaftlichen Problemae oder der Anreizwirkung verfassungsrechtlicher Entscheidungen auf das Verhalten der politischen Akteure“ fehlen (Scharpf 2006: 329). Das Verfassungsgericht ist eben nicht dafür gedacht, eigenständig Politik zu betreiben.

Auch die repräsentativ ausgehandelten expliziten Verfassungsänderungen sind im Hinblick auf eine normativ anspruchsvolle Verfassungstheorie problematisierbar. Denn während die Legitimation des Verfassungsstaates gemeinhin auf die Autorität des bürgerschaftlich verfassten Volkes als Repräsentant seiner selbst zurückgeführt wird, debattierten die meisten Grundgesetzänderungen nur die formal Beteiligten und die direkt Betroffenen jenseits öffentlicher Aufmerksamkeit. Dadurch werden mögliche Argumente dem Diskurs über den Bedarf von Anpassungen an veränderte Rahmenbedingungen, die Angemessenheit von Lösungen und policy learning gar nicht zugeführt und ein Großteil der Gesellschaft nicht in die Verfassungsentwicklung einbezogen. Dies ist kein bloßer Kommentar zur Realitätsnähe einer ohnehin theoretisch gedachten Rechtsfigur, sondern ein Verweis darauf, dass die heutige hohe Akzeptanz des Grundgesetzes angesichts zunehmender „Unübersichtlichkeit" aufgrund von sozioökonomischer Heterogenität, der Aufweichung von Staatsgrenzen und der Schwierigkeit, notwendigermaßen komplexe Entscheidungen den Bürgerinnen und Bürgern zu vermitteln, nicht als selbstverständlich angesehen werden sollte. Die Frage danach, welche Werte die bundesdeutsche Gesellschaft zusammenhalten und welchen Status die Verfassung dabei hat, wird heute so vielfältig beantwortet (Lammert 2006, siehe auch den Beitrag Krischs in diesem Band), dass der Konsens wohl eher in der Vielfalt besteht. Ein solcher Konsens ist potenziell fragil und muss

15 Ein Beispiel ist der Art. 23 (1) GG, der wörtlich Formulierungen aus dem Maastricht-Urteil enthält; Glaeßner 2006: 359. 
immer wieder über Diskurs und Aushandlungen erneuert werden. Mehr Öffentlichkeit zu Ordnung und Wandel des Grundgesetzes könnten zu einer solchen steten Selbstvergewisserung als Verfassungsgemeinschaft beitragen.

Generell sollte nicht mehr oder weniger Verfassungswandel das Ideal sein, sondern die Erhöhung der Konsens- und Rechtfertigungsanforderungen, die Verlängerung der Zeithorizonte in der Verfassungspolitik und wenigstens bei manchen Materien eine erhöhte Partizipation (Grimm 1994: 430; Offe 2003: 13; Schmitter 2003). Die übergeordnete Frage, welche politische Ordnung für die Gesamtgesellschaft die beste ist, und die normative Kohärenz der Verfassung würden so einen höheren politischen und gesellschaftlichen Stellenwert erlangen als in der oft eher kurzfristig, auf einzelne Verfassungsmaterien und die Interessen der Aushandlungsbeteiligten abstellenden aktuellen Verfassungspolitik.

\section{Literatur}

Abromeit, Heidrun (1992): Der verkappte Einheitsstaat, Opladen.

Ackermann, Rolf (2001): Pfadabhängigkeit, Institutionen und Regelreform, Tübingen. Almond, Gabriel A./Sidney Verba (1963): Political Attitudes and Democracy in Five Nations, Princeton.

Batt, Helge-Lothar (1996): Die Grundgesetzreform nach der deutschen Einheit. Akteure, politischer Prozeß und Ergebnisse, Opladen.

Benz, Wolfgang (2005): Zwei Staatsgründungen auf deutschem Boden, in: Deutschland 1945-1949. Bonn: Bundeszentrale für politische Bildung 2005 (= Informationen zur politischen Bildung, Heft 259, in: <http://www.bpb.de/publikationen/ UXE1SE,2,0,Zwei_Staatsgr\%FCndungen_auf_deutschem_Boden.html\#art2> (Zugriff: 30.06 .2008 ).

Benz, Arthur (2005): Kein Ausweg aus der Politikverflechtung? - Warum die Bundesstaatskommission scheiterte, aber nicht scheitern musste, in: Politische Vierteljahresschrift, 46. Jg., H. 2, S. 207-217.

Beyme, Klaus von (1997): Der Gesetzgeber. Der Bundestag als Entscheidungszentrum, Opladen.

Biermann, Kai (2008a): Vertraulichkeit geht vor, in: Die Zeit 9/2008, in: <http://www. zeit.de/online/2008/09/online-durchsuchung-urteil> (Zugriff: 30.06.208).

Biermann, Kai (2008b): Kein Anschluss unter dieser Nummer, in: Die Zeit 13/2008, in: <http://www.zeit.de/online/2008/13/vorratsdaten> (Zugriff: 30.06.2008).

Birthler, Marianne (1995): Lebensfragen, Schule und Pluralität, in: Ansgar Klein (Hrsg.), Grundwerte in der Demokratie, Bonn, S. 164-166.

Böckenförde, Ernst-Wolfgang (1998): Anmerkungen zum Begriff Verfassungswandel, in: Bernd Guggenberger/Thomas Würtenberger (Hrsg.), Hüter oder Lenker der Politik? Das Bundesverfassungsgericht im Widerstreit, Baden-Baden, S. 44-56.

Böckenförde, Ernst-Wolfgang (2000): Verfassungsgerichtsbarkeit. Strukturfragen, Organisation, Legitimation, in: ders. (Hrsg.), Staat, Nation, Europa. Studien zur Staatslehre, Verfassungstheorie und Rechtsphilosophie, 2. Aufl., Frankfurt a.M., S. 157-182.

Bos, Ellen (2004): Verfassungsgebung und Systemwechsel. Die Institutionalisierung von Demokratie im postsozialistischen Osteuropa, Wiesbaden. 
Bryde, Brun-Otto (1982): Verfassungsentwicklung. Stabilität und Dynamik im Verfassungsrecht der Bundesrepublik Deutschland, Baden-Baden.

Burkhart, Simone/Philip Manow (2006): Was bringt die Föderalismusreform? Wahrscheinliche Effekte der geänderten Zustimmungspflicht. MPIfG Working Paper 06/6 des Max-Planck-Instituts für Gesellschaftsforschung, Köln.

Busch, Andreas (1999): Das oft geänderte Grundgesetz, in: Wolfgang Merkel/Andreas Busch (Hrsg.), Demokratie in Ost und West, Frankfurt a.M., S. 549-574.

Busch, Andreas (2006): Verfassungspolitik: Stabilität und permanentes Austarieren, in: Manfred G. Schmidt/Reimut Zohlnhöfer (Hrsg.), Regieren in der Bundesrepublik Deutschland, Wiesbaden, S. 33-56.

BVerfG, 1 BvR 370/07 vom 27.2.2008, Absatz-Nr. (1 - 333), in: <http://www.bverfg. de/entscheidungen/rs20080227 1bvr037007.html > (Zugriff: 30.06.2008).

Clemens, Thomas (1995): Das Bundesverfassungsgericht im Rechts- und Verfassungsstaat, in: Michael Piazolo (Hrsg.), Das Bundesverfassungsgericht. Ein Gericht im Schnittpunkt von Recht und Politik, Mainz, S. 13-31.

Dästner, Christian (2001): Zur Entwicklung der Zustimmungsbedürftigkeit von Bundesgesetzen seit 1949, in: Zeitschrift für Parlamentsfragen, 32. Jg., H. 2, S. 290309.

FAZ [Frankfurter Allgemeine Zeitung], 01.05.2008: Verfassungsrichter Voßkuhle: „Nicht alles zur Frage der Menschenwürde deklarieren“, in: <http://www.faz.net/ s/RubD5CB2DA481C04D05AA471FA88471AEF0/Doc EA2E9FFF86366415D 93A7520F9B0BDD39 ATpl Ecommon Scontent.html> (Zugriff: 30.06.2008)

Feldkamp, Michael F. (1998): Der Parlamentarische Rat 1948-1949. Die Entstehung des Grundgesetzes, Göttingen.

Glaeßner, Gert-Joachim (1977): Herrschaft durch Kader. Leitung der Gesellschaft und Kaderpolitik in der DDR, Opladen.

Glaeßner, Gert-Joachim (1989): Die andere deutsche Republik: Gesellschaft und Politik in der DDR, Opladen.

Glaeßner, Gert-Joachim (1999): Demokratie und Politik in Deutschland, Opladen.

Glaeßner, Gert-Joachim (2003): Sicherheit in Freiheit. Die Schutzfunktion des demokratischen Staates und die Freiheit der Bürger, Opladen.

Glaeßner, Gert-Joachim (2006): Politik in Deutschland, 2.Aufl., Opladen.

Grimm, Dieter (1994): Die Zukunft der Verfassung, 2. Aufl., Frankfurt a.M.

Grimm, Dieter (2005): Die gescheiterte Reform, in: Humboldt-Forum Recht 1/2005, S. $1-5$.

Grundgesetz für die Bundesrepublik Deutschland vom 23. Mai 1949, zuletzt geändert am 28. August 2006, URL: http://www.verfassungen.de/de/gg49-i.htm (Zugriff: 12.09.2006) [mit Nachweis aller Änderungen im Wortlaut].

Große Hüttmann, Martin (1999): Die föderale Staatsform in der Krise? Die öffentliche Debatte um den Föderalismus in Deutschland, in: Der Bürger im Staat, 49. Jg., H. 1/2, S. 107-113.

Grotz, Florian (2009): Verfassungsreformen in der Bundesrepublik: 1969 - 1994 2006, in: Christoph Hönnige/Sascha Kneip/Astrid Lorenz (Hrsg.), Verfassungswandel im Mehrebenensystem, Wiesbaden (i.E.).

Guggenberger, Bernd/Thomas Würtenberger (Hrsg.) (1998): Hüter oder Lenker der Politik? Das Bundesverfassungsgericht im Widerstreit, Baden-Baden.

Henne, Thomas (2006): „Smend oder Hennis“ - Bedeutung, Rezeption und Problematik der ,Lüth-Entscheidung des Bundesverfassungsgerichts von 1958, in: Michael 
Becker/Ruth Zimmerling (Hrsg.), Politik und Recht (= PVS-Sonderheft 36), Wiesbaden, S. 141-150.

Höffe, Otfried (1999): Wieviel Politik ist dem Verfassungsgericht erlaubt?, in: Der Staat, 38. Jg., H. 2, S. 171-193.

Hönnige, Christoph (2007): Verfassungsgericht, Regierung und Opposition. Eine vergleichende Analyse eines Spannungsdreiecks, Wiesbaden.

Hohmann-Dennhardt, Christine (2006): Das Bundesverfassungsgericht und die Frauen, in: Robert C. van Ooyen/Martin H. W. Möllers (Hrsg.), Das Bundesverfassungsgericht im politischen System, Wiesbaden, S. 253-267.

Isensee, Josef (1995a): Die Verfassungsgerichtsbarkeit zwischen Recht und Politik, in: Michael Piazolo (Hrsg.), Das Bundesverfassungsgericht. Ein Gericht im Schnittpunkt von Recht und Politik, Mainz, S. 49-59.

Isensee, Josef (1995b): Die Normativität der Verfassung und der politische Prozeß, in: Adolf Kimmel (Hrsg.), Verfassungen als Fundament und Instrument der Politik, Baden-Baden, S. 25-44.

Kleßmann, Christoph (1991): Die doppelte Staatsgründung. Deutsche Geschichte 1945-1955, 5. Aufl., Bonn.

Kneip, Sascha (2009): Spiel über Bande. Intendierter und nicht-intendierter Verfassungswandel durch Klagen vor dem Bundesverfassungsgericht, in: Christoph Hönnige/Sascha Kneip/Astrid Lorenz (Hrsg.), Verfassungswandel im Mehrebenensystem, Wiesbaden (i.E.).

Korioth, Stefan (2006): Die Rechtsprechung des Bundesverfassungsgerichts zum Bundesstaat, in: Robert C. van Ooyen/Martin H. W. Möllers (Hrsg.), Das Bundesverfassungsgericht im politischen System, Wiesbaden, S. 391-405.

Kruis, Konrad (1995) Das Urteil des Bundesverfassungsgerichts zum Vertrag von Maastricht, in: Michael Piazolo (Hrsg.), Das Bundesverfassungsgericht. Ein Gericht im Schnittpunkt von Recht und Politik, Mainz, S. 125-140.

Lammert, Norbert (Hrsg.) (2006): Verfassung. Patriotismus. Leitkultur. Was unsere Gesellschaft zusammenhält, Bonn.

Landfried, Christine (1984): Bundesverfassungsgericht und Gesetzgeber. Wirkung der Verfassungsrechtsprechung auf parlamentarische Willensbildung und soziale Realität, Baden-Baden.

Landfried, Christine (1994): The Judicialization of Politics in Germany, in: International Political Science Review, 15. Jg., H. 20, S. 113-124.

Lehmbruch, Gerhard (2002) Der unitarische Bundesstaat in Deutschland: Pfadabhängigkeit und Wandel, Discussion Paper des Max-Planck-Instituts für Gesellschaftsforschung, Nr. 2, Köln.

Leutheusser-Schnarrenberger, Sabine (2006): Verfassungspatriotismus als Ziel politischer Bildung, in: <http://www.metatag.de/webs/fdp/sls/files/docs/Patriotismus FAZ.doc> (Zugriff: 30.06.2008).

Limbach, Jutta (1999): Im Namen des Volkes. Macht und Verantwortung der Richter, Stuttgart.

Lorenz, Astrid (2001): Vorwärts in die Vergangenheit? Der Wandel der politischen Institutionen in der Republik Belarus' seit 1991, in: <http://edoc.huberlin.de/ abstract.php3/dissertationen/lorenz-astrid-2001-05-09> (Zugriff: 30.06.2008).

Lorenz, Astrid (2007): Föderalismusreform \& Co. Warum ändert sich das Grundgesetz?, Berlin. 
Lorenz, Astrid (2008): Verfassungsänderungen in etablierten Demokratien. Motivlagen und Aushandlungsmuster, Wiesbaden.

Lorenz, Astrid/Wenke Seemann (2008): Verfassungspolitische Konjunkturzyklen? Überlegungen zur Wirkungsweise konstitutioneller Rigidität, in: Thomas Bräuninger/Joachim Behnke/Susumo Shikano (Hrsg.), Jahrbuch für Handlungsund Entscheidungstheorie Bd. 5, Wiesbaden, 55-86.

Mayer, Franz C. (2002): Deutschland, in: ders. (Hrsg.), Verfassungen in Europa. Ein Überblick, S. 5-10. WHI-Paper 7/2002, in: <http://www.whi-berlin.de/verfassungen. htm> (Zugriff: 30.06.2008).

Mayer, Franz C. (2009): Verfassungswandel durch Annäherung? Der Europäische Gerichtshof, das Bundesverfassungsgericht und das Grundgesetz, in: Christoph Hönnige/Sascha Kneip/Astrid Lorenz (Hrsg.), Verfassungswandel im Mehrebenensystem, Wiesbaden (i.E.).

Merkel, Wolfgang/Eberhard Sandschneider/Dieter Segert (Hrsg.) (1996): Systemwechsel 2. Die Institutionalisierung der Demokratie, Opladen, S. 179-211.

Merritt, Anna J./Richard L. Merritt (Hrsg.) (1970): Public Opinion in Occupied Germany. The OMGUS Surveys, 1945-1949, Urbana (Ill.) u.a.

Merseburger, Peter et al. (1998): Podiumsdiskussion: „Neue Wege. Das Grundgesetz für die alte und die neue Bundesrepublik Deutschland“, in: Dieter Dowe (Hrsg.), Lernen aus der Vergangenheit!? Der Parlamentarische Rat und das Grundgesetz, Bonn.

Mommsen, Hans (1998): Lehren aus der Geschichte der Weimarer Republik bei der Demokratiegründung des Parlamentarischen Rates 1948/49, in: Dieter Dowe (Hrsg.), Lernen aus der Vergangenheit!? Der Parlamentarische Rat und das Grundgesetz, Bonn.

Niclauß, Karlheinz (1998): Der Weg zum Grundgesetz. Demokratiegründung in Westdeutschland 1945-1949, Paderborn.

Offe, Claus (2003): Einleitung. Reformbedarf und Reformoptionen der Demokratie, in: ders. (Hrsg.), Demokratisierung der Demokratie. Diagnosen und Reformvorschläge, Frankfurt a.M., S. 9-23.

Ooyen, Robert C. van (2008): Die Staatstheorie des Bundesverfassungsgerichts und Europa. Von Solange über Maastricht zum EU-Haftbefehl. 2. Aufl., Baden-Baden.

Pfetsch, Frank R. (1990): Ursprünge der zweiten Republik. Prozesse der Verfassungsgebung in den Westzonen und der Bundesrepublik, Opladen.

Piazolo, Michael (1995): Zur Mittlerrolle des Bundesverfassungsgerichts in der deutschen Verfassungsordnung. Eine Einleitung, in: ders. (Hrsg.), Das Bundesverfassungsgericht. Ein Gericht im Schnittpunkt von Recht und Politik, Mainz, S. 7-11.

Preuß, Ulrich K. (1995): Die Verfassung als Wertordnung, in: Ansgar Klein (Hrsg.), Grundwerte in der Demokratie, Bonn, S. 44-47.

Renzsch, Wolfgang (1991): Finanzverfassung und Finanzausgleich. Die Auseinandersetzungen um ihre politische Gestaltung in der Bundesrepublik Deutschland zwischen Währungsreform und deutscher Vereinigung (1948 bis 1990), Bonn.

Reutter, Werner (1998): Politik und Verfassung - Rechts- und politikwissenschaftliche Beiträge zu einem ungeklärten Verhältnis, in: Politische Vierteljahresschrift, 39. Jg., H. 4, S. 858-870.

Reutter, Werner (2001): Das Bundesverfassungsgericht als Teil des politischen Systems der Bundesrepublik Deutschland. Ein politikwissenschaftlicher Interpretationsversuch, in: Gert-Joachim Glaeßner/Werner Reutter/Charlie Jeffery (Hrsg.), 
Verfassungspolitik und Verfassungswandel. Deutschland und Großbritannien im Vergleich, Wiesbaden, S. 99-124.

Scharpf, Fritz W. (2006): Recht und Politik in der Reform des deutschen Föderalismus, in: Michael Becker/Ruth Zimmerling (Hrsg.), Politik und Recht (= PVSSonderheft 36), Wiesbaden, S. 306-332.

Schlaich, Klaus/Stefan Korioth (2004): Das Bundesverfassungsgericht. Stellung, Verfahren, Entscheidungen, München.

Scholz, Rupert (1995): Verfassungswerte und Wertewandel, in: Ansgar Klein (Hrsg.), Grundwerte in der Demokratie, Bonn, S. 40-43.

Schmitter, Philippe C. (2003): Wie könnte eine „postliberale“ Demokratie aussehen? Skizzenhafte Vermutungen und Vorschläge, in: Claus Offe (Hrsg.), Demokratisierung der Demokratie. Diagnosen und Reformvorschläge, Frankfurt a.M. u.a., S. 152-165.

Schuppert, Gunnar Folke (1980): Funktionell-rechtliche Grenzen der Verfassungsinterpretation, Königstein.

Schuppert, Gunnar Folke/Christian Bumke (Hrsg.) (2000): Bundesverfassungsgericht und gesellschaftlicher Grundkonsens, Baden-Baden.

Schwind, Margarete et al. (1999): Das 20. Jahrhundert in Wort, Bild, Film und Ton. Die 40er Jahre, Stuttgart.

Steffani, Winfried (1979): Das präsidentielle System der USA und die parlamentarischen Systeme Großbritanniens und Deutschlands im Vergleich, in: ders., Parlamentarische und präsidentielle Demokratie, Opladen.

Sternberger, Dolf (1982): Verfassungspatriotismus (= Schriften, Bd. 10), Frankfurt a.M.

Stone Sweet, Alec (2000): Governing with Judges. Constitutional Politics in Europe, Oxford.

Stüwe, Klaus (1997): Die Opposition im Bundestag und das Bundesverfassungsgericht. Das verfassungsrechtliche Verfahren als Kontrollinstrument der parlamentarischen Minderheit, Baden-Baden.

Vanberg, Georg (2005): The Politics of Constitutional Review in Germany, Cambridge.

Voßkuhle, Andreas (2004): Gibt es und wozu nutzt eine Lehre vom Verfassungswandel?, in: Der Staat 43. Jg., H. 3, S. 450-459.

Wahl, Rainer (1998): Quo Vadis - Bundesverfassungsgericht? Zur Lage von Verfassungsgerichtsbarkeit, Verfassung und Staatsdenken, in: Bernd Guggenberger/Thomas Würtenberger (Hrsg.), Hüter oder Lenker der Politik? Das Bundesverfassungsgericht im Widerstreit, Baden-Baden, S. 81-120.

Wesel, Uwe (2004): Der Gang nach Karlsruhe. Das Bundesverfassungsgericht in der Geschichte der Bundesrepublik, München. 